\title{
Association of Antimicrobial Susceptibility and Treatment Outcome in Acne Vulgaris Patients: A Pilot Study
}

\author{
Ashvini K. Yadav ${ }^{1}$ Suneel Bhooshan ${ }^{1}$ Allen Johnson ${ }^{1}$ \\ Debasis Biswas ${ }^{1}$ \\ ${ }^{1}$ Department of Microbiology, All India Institute of Medical Sciences \\ (AlIMS), Bhopal, Madhya Pradesh, India \\ 2Department of Dermatology, All India Institute of Medical Sciences \\ (AlIMS), Bhopal, Madhya Pradesh, India
}

Dinesh P. Asati ${ }^{2} \quad$ Shashwati Nema ${ }^{1}$

Address for correspondence Debasis Biswas, MD, Department of Microbiology, AlIMS Bhopal, Bhopal, Madhya Pradesh, 462024, India (e-mail: debasis.microbiology@aiimsbhopal.edu.in).

J Lab Physicians:2020;12:233-238

\begin{abstract}
Keywords

- Cutibacterium acnes

- Acne vulgaris

- Antibigram

- In vitro susceptibility

- Propionibacterium acnes

Purpose Cutibacterium acnes (C. acnes) is an emerging pathogen that is highly resistant to antibiotics and is capable of causing persistent infections that are difficult to treat.

Methods \& Materials Acne vulgaris patients visiting dermatology OPD of our tertiary care hospital during the study period of 2 months were recruited. Skin swabs were collected, and the sample was processed on $5 \%$ sheep-blood agar for anaerobic culture by the GasPak method. Isolates were identified by the standard biochemical test. Antimicrobial susceptibility testing was performed for clinically relevant antibiotics by the E-strip method. The clinical response was evaluated after 1-month follow-up to the prescribed antibiotics.

Results Minocycline, doxycycline, ceftriaxone, and tetracycline were the most effective antibiotics. Nonsusceptibility to clindamycin and erythromycin were observed in $11.9 \%$ and $31 \%$ isolates, respectively, with $9.5 \%$ isolates being nonsusceptible to both. For none of the antibiotics we found significant difference in the proportion of susceptible and nonsusceptible isolates between mild, moderate, and severe grades of acne vulgaris. For none of the antibiotic regimens, significant difference was observed between nonresponders and responders. Twenty-seven patients received clindamycin and among them 16 of 19 responders and 6 of 8 nonresponders yielded growth of clindamycin-susceptible isolates ( $p=0.57$ ).

Conclusion We observed significant prevalence of resistant strains of $C$. acnes among patients with acne vulgaris. No association was observed between in vitro susceptibility results and treatment outcome.
\end{abstract}

\section{Introduction}

Cutibacterium acnes (C. acnes), an anaerobic gram-positive bacillus formerly known as Propionibacterium acnes, is a significant emerging pathogen that has lately been incriminated in the pathogenesis of acne vulgaris as well as life-threatening infections such as endocarditis, intravascular

DOI https://doi.org/ 10.1055/s-0040-1720943 ISSN 0974-2727. infections, post-craniotomy infections, endophthalmitis, and septic arthritis. ${ }^{1-4}$ The involvement of this bacterium is also being increasingly reported in the context of orthopedic and cardiac prosthetic infections, infections of breast implants, intraocular lenses, and ventriculoperitoneal shunts. ${ }^{5}$

It has been the most classical and consistent pathogen associated with acne vulgaris. ${ }^{6}$ This organism is abundant in 
sebum-rich areas (which are prone to acne), causes blockage of pilosebaceous glands by making biofilms, and also plays an important role in initiation and propagation of inflammation. ${ }^{7}$ Biofilm formation makes them highly resistant to antimicrobial agents and is capable of causing chronic persistent infection that is difficult to treat.

Therapeutically, the most effective antibacterial agents in acne vulgaris target $C$. acnes. ${ }^{6}$ Optimal antibiotic therapy, directed against $C$. acnes, is the key to the treatment of these infections. But the widespread and long-term use of oral and topical Macrolides and Tetracyclines has resulted in significant dissemination of cross-resistant strains of cutibacteria in many parts of the world. Resistant strains have been reported to be widely prevalent throughout Europe, Japan, Korea, Singapore, Australia, and USA.-10

Information regarding the antimicrobial susceptibility profile and resistance mechanisms of $C$. acnes is relatively scarce from India. ${ }^{11}$ Being an anaerobic slow-growing organism, the isolation and antibiotic sensitivity testing of this bacterium are technically challenging tasks that require rapid transport of samples to the microbiology laboratory; use of special transport media; infrastructure for performing anaerobic culture; skilled manpower; and relatively lengthy incubation periods. Consequently, isolation and characterization of this organism is seldom undertaken in clinical microbiology laboratories.

In view of the paucity of data in the Indian context and the need to tailor antibiotic therapy according to local antibiogram patterns, the present study was conducted with the objective of analyzing a repertoire of clinical isolates of C. acnes from patients afflicted with acne vulgaris, evaluating the outcome in these patients following standard therapy with prescribed antibiotics, and delineating the association between clinical response and in vitro susceptibility of the recovered isolates with clinically relevant antibiotics.

\section{Materials and Methods}

\section{Ethical Considerations}

The study protocol was approved by the institutional human ethics committee, and informed consent was obtained from each patient prior to recruitment.

Consecutive patients, attending the Dermatology OPD of a tertiary care teaching hospital in Central India with acne vulgaris, were recruited into the study over a period of 2 months. Patients already receiving antibiotic treatment at the time of presentation or within the last 4 weeks were excluded from the study. Relevant demographic and clinical details of the recruited patients were recorded in a predesigned case study form. The treatment given to the patients, following diagnostic workup, was also recorded. The patients were called for follow- up at the end of 1 month during which the outcome of treatment was recorded.

A single skin swab was collected from each recruited participant for anaerobic culture of $C$. acnes. The sample from acne lesions was collected by applying firm pressure and rubbing against the lesions with a transport swab. The swab was moistened in sodium phosphate buffer $(0.075 \mathrm{~mol} / \mathrm{L}$; $\mathrm{pH} 7.9$ ) containing $0.1 \%$ Triton-X 100 . After specimen collection, the swabs were immediately transported to the microbiology laboratory where they were cultured in 5\% sheep blood agar under anaerobic conditions. The swabs were gently rolled over the surface of a portion of the plate and then streaked to obtain isolated colonies. The anaerobic incubation was done using the GasPak Anaerobic System of GasPaks (HiMedia, Mumbai) at $37^{\circ} \mathrm{C}$ for $72 \mathrm{hrs}$. Colonies of C. acnes were identified among the recovered isolates using phenotypic features such as cultural characteristics, microscopic morphology, and standard biochemical tests. ${ }^{12}$ The primary isolate of $C$. acnes was then subcultured in 5\% sheep blood agar to obtain a pure growth of $C$. acnes, which was then analyzed to determine the minimum inhibitory concentration (MIC) of a range of clinically relevant antibiotics using commercially procured E-strips. Inoculum for the susceptibility studies were prepared by suspending a culture grown for 48 hours in reduced Brucella broth to achieve a final concentration equivalent to a $0.5 \mathrm{McF}$ arland standard $\left(1.5 \times 10^{8} \mathrm{CFU} / \mathrm{ml}\right)$. E-test strips of each of the test antibiotics were then placed onto the Brucella blood agar supplemented with Vit.K1, hemin and laked sheep blood, and the plates were incubated at $37^{\circ} \mathrm{C}$ under anaerobic conditions ${ }^{13}$. MICs for each antibiotic were determined after 48 hours of incubation.

Antibiotic susceptibility and resistance breakpoints were defined as follows

\begin{tabular}{|l|l|}
\hline Antibiotic agent & Clinical breakpoint \\
\hline Clindamycin & $\geq 8 \mu \mathrm{g} / \mathrm{mL}^{13}$ \\
\hline Tetracycline & $\geq 16 \mu \mathrm{g} / \mathrm{mL}^{13}$ \\
\hline Ceftriaxone & $\geq 64 \mu \mathrm{g} / \mathrm{mL}^{13}$ \\
\hline Ertapenem & $\geq 16 \mu \mathrm{g} / \mathrm{mL}^{13}$ \\
\hline Meropenem & $\geq 16 \mu \mathrm{g} / \mathrm{mL}^{13}$ \\
\hline Erythromycin & $\geq 2 \mu \mathrm{g} / \mathrm{mL}^{11}$ \\
\hline Doxycycline & $\geq 4 \mu \mathrm{g} / \mathrm{mL}^{11}$ \\
\hline Minocycline & $\geq 16 \mu \mathrm{g} / \mathrm{mL}^{11}$ \\
\hline
\end{tabular}

Patients were evaluated during their follow-up visits after 1 month, and the clinical response to the prescribed antibiotics was recorded.

\section{Statistics}

To understand the association between in vitro resistance and treatment outcome, we compared the cure rates between patients yielding sensitive and resistant isolates of $C$. acnes. Chisquare test was used to compare cure rates between patients with susceptible and nonsusceptible isolates, and the $p$ value was calculated with the help of the Epi Info online software.

\section{Results}

Skin swabs from a total of 52 consecutively recruited patients attending skin OPD with acne vulgaris were collected for 
C. acnes culture. Growth of $C$. acnes was observed in 42 samples $(80.76 \%)$. These 42 patients were included for further analysis and follow-up.

The mean ( \pm standard deviation [SD]) age of the patients was $21( \pm 4)$ years and the mean $( \pm$ SD) duration of disease was $3( \pm 2)$ years. Thirty-one patients presented with acne only on their face, two presented with acne only on their trunk, and nine patients had lesions both on face and trunk. Acne was mild in intensity in 8 patients, moderate in 25 patients, and severe in 9 patients. The lesions were inflammatory in 9 patients, noninflammatory in 7 patients, and of mixed type in 26 patients. Seven patients reported the use of oil-based cosmetics on their face and three patients gave a history of having facial massages. The patients received treatment according to their clinical presentation from a single qualified dermatologist and were followed-up after 1 month of treatment. They were categorized into nonresponder $(n=16)$ and responder $(n=26)$ groups, depending on improvement or deterioration with the prescribed treatment. The two subgroups of patients were found to be comparable with respect to their age and gender distribution, clinical characteristics, and nature of treatment administered ( - Table $\mathbf{1}$ ).

Colonies of $C$. acnes were small (less than $0.5 \mathrm{~mm}$ in size), grey, and weakly $\beta$ hemolytic (-Fig. 1a). On microscopy after Gram staining, nonsporing Gram-positive bacilli were seen (inset image of $\boldsymbol{- F i g}$. 1a). These colonies were confirmed to be $C$. acnes by performing standard biochemical tests. ${ }^{11}$ Antibiotic susceptibility testing with clinically relevant antibiotics was performed; minocycline (100\% susceptible), doxycycline (97.6\% susceptible), ceftriaxone (95.2\% susceptibility), and tetracycline (92.9\% susceptibility) were found to be the most effective antibiotics. Nonsusceptibility to clindamycin and erythromycin was observed in $11.9 \%$ $(n=5)$ and $31 \%(n=13)$ isolates, respectively, with $9.5 \%(n=4)$ isolates being nonsusceptible to both. For all the antibiotics tested, a significantly higher proportion of isolates was susceptible; Ertapenem was found to be the least effective antibiotic ( $52.4 \%$ susceptibility) (-Table 2 ).

On examining the antibiotics prescribed for these patients, we found a total of five regimens being administered in 35 patients. Antibiotics were not administered in 7 patients, while 19 patients received only topical antibiotics. Sixteen patients were given erythromycin $(n=14)$ and
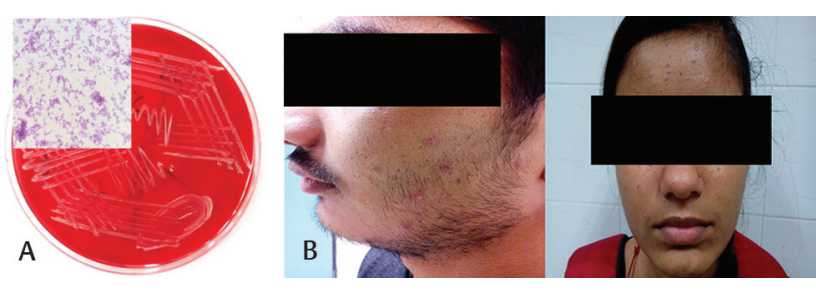

Fig. 1 (a) C. acnes colonies on blood agar plate, inset image showing gram staining. (b) Clinical representation of acne on cheek and forehead area.

Table 1 Comparison of age and gender distribution, clinical characteristics, and nature of treatment administered among nonresponders and responders

\begin{tabular}{|l|l|l|l|}
\hline Parameters & Nonresponders $(\boldsymbol{n}=16)$ & Responders $(\boldsymbol{n}=\mathbf{2 6})$ & $\boldsymbol{p}$-Value \\
\hline Age (years) (mean \pm SD) & $22.12 \pm 5.95$ & $21 \pm 2.92$ & 0.00169 \\
\hline Gender (M/F) & $13 / 03$ & $20 / 06$ & 0.7400 \\
\hline Duration of illness (months) (mean \pm SD) & $42 \pm(31.58)$ & $28.58 \pm(22.68)$ & 0.1386 \\
\hline Facial involvement (\%) & $16(100)$ & $05(19.23)$ & 0.3883 \\
\hline Extrafacial involvement (\%) & $06(37.50)$ & $04: 17: 05$ & 0.3883 \\
\hline Grade of illness (mild:moderate:severe) & $04: 08: 04$ & $20(76.92)$ & 0.598 \\
\hline Inflammation present (\%) & $15(93.75)$ & $10(38.46)$ & 0.1554 \\
\hline Only topical antibiotic administered (\%) & $03(18.75)$ & $16(61.54)$ & 0.2701 \\
\hline Systemic antibiotic administered (\%) & $13(81.25)$ & $10(38.46)$ & 0.3393 \\
\hline History of previous treatment (\%) & $08(50)$ & 0.4631 \\
\hline
\end{tabular}

Table 2 Antibiotic susceptibility pattern of C. acnes isolates $(n=42)$

\begin{tabular}{|l|l|l|l|}
\hline Name of Antibiotics & Susceptible & Nonsusceptible & $p$-Value \\
\hline Clindamycin & $37(88.10 \%)$ & $5(11.90 \%)$ & $<0.001$ \\
\hline Tetracycline & $39(92.86 \%)$ & $3(7.14 \%)$ & $<0.001$ \\
\hline Ceftriaxone & $40(95.24 \%)$ & $2(4.76 \%)$ & $<0.001$ \\
\hline Ertapenem & $22(52.38 \%)$ & $20(47.62 \%)$ & $<0.001$ \\
\hline Meropenem & $25(59.52 \%)$ & $17(40.48 \%)$ & $<0.001$ \\
\hline Erythromycin & $29(69.05 \%)$ & $13(30.95 \%)$ & $<0.001$ \\
\hline Doxycycline & $41(97.62 \%)$ & $1(2.38 \%)$ & $<0.001$ \\
\hline Minocycline & $42(100 \%)$ & 0 & $<0.001$ \\
\hline
\end{tabular}


doxycycline $(n=2)$ in addition to topical clindamycin. For none of the five regimens, significant difference was observed between the proportion of nonresponders and responders (-Table 3), implying that none of the regimens could claim superiority over others.

We then tried to analyze if there was a difference in the proportion of susceptible and nonsusceptible isolates between mild, moderate, and severe categories of patients. For none of the antibiotics, we found significant difference in the proportion of susceptible and nonsusceptible isolates among the three categories of disease severity. Thus, antibiotic susceptibility of $C$. acnes was not found to be associated with its virulence and pathogenic potential (-Table 4).

We next tried to find an association between the clinical response of the patients and antibiotic susceptibility of the recovered isolates. Among the 35 patients who received antibiotics, 27 patients received clindamycin, 16 patients received erythromycin and 8 patients received doxycycline, either alone or in combination. It was observed that of the 27 patients who received clindamycin, 16 of the 19 responders and 6 of the 8 nonresponders yielded growth of clindamycin-susceptible isolates $(p=0.57)$.

\section{Discussion}

In the present study on the antibiogram of the emerging pathogen, $C$. acnes, it was observed that minocycline, doxycycline, ceftriaxone, and tetracycline were the most effective antibiotics and carbapenems were the least effective. Nonsusceptibility to clindamycin was observed in $11.9 \%$ of the isolates, and no association could be drawn between clindamycin susceptibility and treatment response.
Similar to our findings, other authors from different parts of the world have also reported consistently decreasing susceptibility to clindamycin and erythromycin among C. acnes isolates. clindamycin susceptibility has been reported to vary from 7.5 to $91 \%$ and erythromycin susceptibility from 10.4 to $98 \%$ in different studies. ${ }^{14-26}$ It is worth noting that development of antibiotic resistance in $C$. acnes was not encountered until the early 1980s despite years of systemic use of tetracycline and erythromycin. But shortly after the introduction of topical formulation of these drugs, the first strains of resistant $C$. acnes emerged in the USA, and by the late 1980s, very high MIC levels for these drugs became commonplace among $C$. acnes isolates from UK and USA. ${ }^{8}$ Several other studies have also demonstrated similar relationship between the increasing resistance to these drugs and clinical prescription patterns. In a study from Singapore, Tan et $\mathrm{al}^{10}$ have shown resistance to erythromycin to be more significantly associated with increased duration of antibiotic usage. Patients on no antibiotics or on short-term antibiotics (6-18 weeks) were found to yield much lesser isolation of erythromycin-resistant strains, and most of the erythromycin-resistant strains were also cross-resistant to clindamycin. In a larger study covering six European centers, widespread use of topical erythromycin and clindamycin was found to cause significant dissemination of cross-resistant strains of Cutibacteria both in patients and in their untreated contacts. This study also revealed remarkable variations in the proportion of resistant Cutibacteria between the European countries, which was reflective of the usage pattern in these countries. Interestingly, a significant proportion of dermatologists specializing in acne treatment were found to be colonized with resistant Cutibacteria in this study, while none of the

Table 3 Antibiotics regimens prescribed to patients $(n=35)$

\begin{tabular}{|l|l|l|l|l|}
\hline Antibiotics regimens & Total & Nonresponders & Responders & $p$-Value \\
\hline Erythromycin + Clindamycin & 14 & $5(35.71 \%)$ & $9(65.29 \%)$ & 0.6726 \\
\hline Doxycycline + Clindamycin & 2 & $1(50 \%)$ & $1(50 \%)$ & 0.7662 \\
\hline Erythromycin & 2 & 2 & 0 & 0.07447 \\
\hline Doxycycline & 6 & $4(66.67 \%)$ & $2(33.33 \%)$ & 0.9569 \\
\hline Clindamycin & 11 & $2(18 \%)$ & $9(72 \%)$ & 0.07447 \\
\hline
\end{tabular}

Table 4 Antibiotics susceptibility pattern of C. acnes isolates in different categories of disease severity

\begin{tabular}{|l|l|l|l|l|l|l|l|}
\hline \multirow{2}{*}{$\begin{array}{l}\text { Name of } \\
\text { Antibiotics }\end{array}$} & \multicolumn{2}{|l|}{ Mild (08) } & Moderate (25) & \multicolumn{2}{l|}{ Severe (09) } \\
\cline { 2 - 7 } & Susceptible & Nonsusceptible & Susceptible & Nonsusceptible & Susceptible & Nonsusceptible \\
\hline Clindamycin & $7(87.50 \%)$ & $1(12.50 \%)$ & $22(88.00 \%)$ & $3(12.00 \%)$ & $8(88.89 \%)$ & $1(11.11 \%)$ & 0.9958 \\
\hline Tetracycline & $7(87.50 \%)$ & $1(12.50 \%)$ & $24(96.00 \%)$ & $24(96.00 \%)$ & $8(88.89 \%)$ & $1(11.11 \%)$ & 0.6275 \\
\hline Ceftriaxone & $7(87.50 \%)$ & $1(12.50 \%)$ & $24(96.00 \%)$ & $24(96.00 \%)$ & $8(88.89 \%)$ & $1(11.11 \%)$ & 0.6275 \\
\hline Ertapenem & $3(37.50 \%)$ & $5(62.50 \%)$ & $11(44.00 \%)$ & $14(56.00 \%)$ & $5(55.56 \%)$ & $4(44.44 \%)$ & 0.7425 \\
\hline Meropenem & $5(62.50 \%)$ & $3(37.50 \%)$ & $14(56.00 \%)$ & $11(44.00 \%)$ & $6(66.67 \%)$ & $3(33.33 \%)$ & 0.8399 \\
\hline Erythromycin & $7(87.50 \%)$ & $1(12.50 \%)$ & $17(68.00 \%)$ & $8(32.00 \%)$ & $5(55.56 \%)$ & $4(44.44 \%)$ & 0.3581 \\
\hline Doxycycline & $7(87.50 \%)$ & $1(12.50 \%)$ & $25(100.00 \%)$ & $0(00.00 \%)$ & $9(100.00 \%)$ & $0(00.00 \%)$ & 0.1134 \\
\hline Minocycline & $8(100 \%)$ & $0(0.00 \%)$ & $25(100.00 \%)$ & $0(00.00 \%)$ & $9(100.00 \%)$ & $0(00.00 \%)$ & - \\
\hline
\end{tabular}


nondermatologist physicians were similarly colonized. ${ }^{27}$ In a recent study from Korea too, increasing resistance to clindamycin and erythromycin has been reported among $C$. acnes isolates from acne patients, compared to previous studies from the same region. ${ }^{28}$ In sync with these studies, our finding of a relatively high proportion of clindamycin nonsusceptibility could be explained by virtue of its being the most commonly prescribed antibiotic among our patients.

In the event of the rising trend of resistance to clindamycin and erythromycin, alternative therapeutic choices against this emerging pathogen need to be explored. In contrast to the obvious advantages of topical formulation for skin lesions, systemic administration of antibiotics is imperative in C. acnes isolates recovered from deep-seated infections such as endocarditis, septic arthritis, endophthalmitis, etc. and diverse device- and implant-related infections. Among the systemic choices, our finding regarding minocycline, doxycycline, ceftrioxone and tetracyclines being the most effective antibiotics is also corroborated by other authors. Universal susceptibility to ceftrioxone has been reported in independent studies from USA and Switzerland. ${ }^{3,26,29}$ In a study from Singapore, susceptibility of $C$. acnes to different tetracyclines was compared and the average MIC to tetracyclines was found to be higher than that of doxycycline which, in turn, was higher than that for minocycline. ${ }^{10}$ In another study from Korea, Song et $\mathrm{al}^{9}$ observed a consumption-related decline in susceptibility toward doxycycline with patients with a history of treatment with topical and systemic antibiotics showing higher MIC to doxycycline. Like other antibiotics, geographical variation has also been noted in doxycycline susceptibility pattern of $C$. acnes, with resistance rates ranging from 7 to $63 \%$ being reported in literature. We observed considerably higher proportion of resistance among the recovered $C$. acnes isolates toward carbapenems. Contrary to our findings, Shame et $\mathrm{al}^{3}$ and Crane et $\mathrm{a}^{26}$ have reported all the isolates to be within the susceptible range of MIC for ertapenem and meropenem in their studies conducted in 2006 and 2013, respectively. This difference with our findings could be explained in view of geographical and temporal variation and also by relatively unrestricted use of carbapenems in Indian clinical practice.

We observed the lack of association between clindamycin susceptibility and treatment outcome in our study. This difference in in vitro result and in vivo response could partially be explained by the known propensity of this bacterium to produce biofilms at the site of clinical lesions. ${ }^{29}$ This ability to produce biofilm could be responsible for offering protection to the bacteria from the inhibitory effect of the administered antibiotics. Pharmacokinetic factors like subinhibitory concentration of the antibiotics at the site of infection could also be responsible for inadequate treatment outcome. Moreover, the requirement of alkaline $\mathrm{pH}$ for antibacterial action of clindamycin and erythromycin could also have remained unmet at the site of clinical lesions. ${ }^{30}$

The present study has two major limitations. First, the sample size in this study was relatively small and as a result, adequate representation of the different treatment regimens could not be achieved. Second, the molecular mechanism of resistance to the different antibiotics could not be included in the scope of the study. Building on the findings of this pilot study, we are planning to conduct a broader and more elaborate study on clinico-microbiological and molecular characteristics of antibiotic susceptibility in $C$. acnes isolates recovered at our institute.

\section{Conclusion:}

Our pilot study brings out the antibiogram of clinical isolates of C. acnes in the Indian context and records a lack of association between the antibiotic susceptibility of these isolates with the clinical response to treatment. More elaborate studies need to be undertaken to validate our findings and identify the determinants of treatment outcome targeting this emerging pathogen.

\section{Conflicts of Interest}

None declared.

\section{References}

1 Mohsen AH, Price A, Ridgway E, West JN, Green S, McKendrick MW. Propionibacterium acnes endocarditis in a native valve complicated by intraventricular abscess: a case report and review. Scand J Infect Dis 2001; 33(5):379-380

2 Kelly ME, Fourney DR, Guzman R, Sadanand V, Griebel RW, Sanche SE. Propionibacterium acnes infections after cranial neurosurgery. Can J Neurol Sci 2006;33(3):292-295

3 Shames R, Satti F, Vellozzi EM, Smith MA. Susceptibilities of Propionibacterium acnes ophthalmic isolates to ertapenem, meropenem, and cefepime. J Clin Microbiol 2006;44(11):4227-4228

4 Yocum RC, McArthur J, Petty BG, Diehl AM, Moench TR. Septic arthritis caused by Propionibacterium acnes. JAMA 1982;248(14):1740-1741

5 Aubin GG, Portillo ME, Trampuz A, Corvec S. Propionibacterium acnes, an emerging pathogen: from acne to implant-infections, from phylotype to resistance. Med Mal Infect 2014;44(6):241-250

6 Platsidaki E, Dessinioti C. Recent advances in understanding Propionibacterium acnes (Cutibacterium acnes) in acne [version 1; referees: 2 approved] F1000Research 2018, 7(F1000 Faculty Rev):1953

7 Bayston R, Ashraf W, Barker-Davies R, et al. Biofilm formation by Propionibacterium acnes on biomaterials in vitro and in vivo: impact on diagnosis and treatment. J Biomed Mater Res A 2007;81(3):705-709

8 Eady EA, Gloor M, Leyden JJ. Propionibacterium acnes resistance: a worldwide problem. Dermatology 2003;206(1):54-56

9 Song M, Seo SH, Ko HC, et al. Antibiotic susceptibility of Propionibacterium acnes isolated from acne vulgaris in Korea. J Dermatol 2011;38(7):667-673

10 Tan HH, Goh CL, Yeo MG, Tan ML. Antibiotic sensitivity of Propionibacterium acnes isolates from patients with 
acne vulgaris in a tertiary dermatological referral centre in Singapore. Ann Acad Med Singapore 2001;30(1):22-25

11 Sardana K, Gupta T, Kumar B, Gautam HK, Garg VK. Cross-sectional pilot study of antibiotic resistance in Propionibacterium acnes strains in Indian acne patients using 16S-RNA polymerase chain reaction: a comparison among treatment modalities including antibiotics, benzoyl peroxide, and isotretinoin. Indian J Dermatol 2016;61(1):45-52

12 Jousimies-Somer HR, Summanem P, Citron DM, Baron EJ, Wexler H, Finegold SM. Wadsworth-KTL Anaerobic Bacteriology Manual. 6th ed. Belmont, CA: Star Publishing Co.; 2002

13 Clinical and Laboratory Standard Institute. Performance Standards for Antimicrobial Susceptibility Testing: TwentyEighty Informational Supplement M100-S28. Wayne, PA: CLSI; 2018

14 Coates P, Vyakrnam S, Eady EA, Jones CE, Cove JH, Cunliffe WJ. Prevalence of antibiotic-resistant propionibacteria on the skin of acne patients: 10-year surveillance data and snapshot distribution study. Br J Dermatol 2002;146(5):840-848 PubMed

15 Oprica C, Emtestam L, Lapins J, et al. Antibiotic-resistant Propionibacterium acnes on the skin of patients with moderate to severe acne in Stockholm. Anaerobe 2004;10(3):155-164

16 Gübelin W, Martínez MA, Molina MT, Zapata S, Valenzuela ME. Antimicrobial susceptibility of strains of Propionibacterium acnes isolated from inflammatory acne. Rev Latinoam Microbiol 2006;48(1):14-16

17 Ergin C, Ergin S, Kaleli I, Kaçar N, Sengül M, Erdoğan BS. Nasal antibiotic-resistant Propionibacterium acnes carriage in acne vulgaris patients in Turkey. J Dermatol 2006;33(12):899-901

18 Tan HH, Tan AW, Barkham T, Yan XY, Zhu M. Communitybased study of acne vulgaris in adolescents in Singapore. $\mathrm{Br} \mathrm{J}$ Dermatol 2007;157(3):547-551

19 González R, Welsh O, Ocampo J, et al. In vitro antimicrobial susceptibility of Propionibacterium acnes isolated from acne patients in northern Mexico. Int J Dermatol 2010;49(9):1003-1007

20 Dumont-Wallon G, Moyse D, Blouin E, Dréno B. Bacterial resistance in French acne patients. Int $\mathrm{J}$ Dermatol 2010;49(3):283-288
21 Zandi S, Vares B, Abdollahi H. Determination of microbial agents of acne vulgaris and Propionibacterium acnes antibiotic resistance in patients referred to dermatology clinics in Kerman, Iran, 2008. Jundishapur J Microbiol 2011;4:17-22

22 Schafer F, Fich F, Lam M, Gárate C, Wozniak A, Garcia P. Antimicrobial susceptibility and genetic characteristics of Propionibacterium acnes isolated from patients with acne. Int J Dermatol 2013;52(4):418-425

23 Luk NM, Hui M, Lee $\mathrm{HC}$, et al. Antibiotic-resistant Propionibacterium acnes among acne patients in a regional skin centre in Hong Kong. J Eur Acad Dermatol Venereol 2013;27(1):31-36

24 Mendoza N, Hernandez PO, Tyring SK, Haitz KA, Motta A. Antimicrobial susceptibility of Propionibacterium acnes isolates from acne patients in Colombia. Int $\mathrm{J}$ Dermatol 2013;52(6):688-692

25 Abdel Fattah NS, Darwish YW. In vitro antibiotic susceptibility patterns of Propionibacterium acnes isolated from acne patients: an Egyptian university hospital-based study. J Eur Acad Dermatol Venereol 2013;27(12):1546-1551

26 Crane JK, Hohman DW, Nodzo SR, Duquin TR. Antimicrobial susceptibility of Propionibacterium acnes isolates from shoulder surgery. Antimicrob Agents Chemother 2013;57(7):3424-3426

27 Ross JI, Snelling AM, Carnegie E, et al. Antibiotic-resistant acne: lessons from Europe. Br J Dermatol 2003;148(3):467-478

28 Moon SH, Roh HS, Kim YH, Kim JE, Ko JY, Ro YS. Antibiotic resistance of microbial strains isolated from Korean acne patients. J Dermatol 2012;39(10):833-837

29 Furustrand Tafin U, Corvec S, Betrisey B, Zimmerli W, Trampuz A. Role of rifampin against Propionibacterium acnes biofilm in vitro and in an experimental foreign-body infection model. Antimicrob Agents Chemother 2012;56(4):1885-1891

30 Deck DH, Winston LG. Tetracyclines, Macrolides, Clindamycin, Chloramphenicol, Streptogramins, \& Oxazolidinones. In: Katzung BG, Trevor AJ, eds. Basic and Clinical Pharmacology. 13th ed. New York, NY: McGraw Hill; 2015:792 